\title{
Magneto-optical properties of nickel nanowire arrays
}

\author{
Sonia Melle, ${ }^{\text {a) }}$ José Luis Menéndez, and Gaspar Armelles \\ Instituto de Microelectrónica de Madrid, CSIC, Tres Cantos, 28760 Madrid, Spain
}

David Navas and Manuel Vázquez

Instituto de Ciencia de Materiales, CSIC, Cantoblanco, 28049 Madrid, Spain

Kornelius Nielsch

Massachusetts Institute of Technology, Department of Materials Science and Engineering, Cambridge, Massachusetts 02139

Ralf B. Wehrspohn ${ }^{\text {b) }}$ and Ulrich Gösele

Max-Planck-Institute of Microstructure Physics, Weinberg 2, 06120 Halle, Germany

(Received 18 July 2003; accepted 7 October 2003)

\begin{abstract}
We report on the magneto-optical properties of hexagonally arranged $\mathrm{Ni}$ nanowires embedded in anodic alumina templates. Due to the nanowire geometry, these samples show different response depending on the polarization orientation of the incident light, which leads to a high anisotropy of both their optical and magneto-optical properties. A strong increase of the magneto-optical activity is clearly observed with respect to the Ni bulk material. We associate this behavior to plasmon resonance of the Ni nanowires. (C) 2003 American Institute of Physics. [DOI: 10.1063/1.1630840]
\end{abstract}

Magnetic nanowire arrays have attracted great interest for their particular magnetic properties with possible applications as perpendicular magnetic storage media. ${ }^{1}$ Although there have been considerable efforts to determine the magnetization behavior of magnetic nanowire arrays, ${ }^{2,3}$ very few studies have been focused on their magneto-optical (MO) properties. ${ }^{4}$ In this work, we report on spectroscopic measurements of the MO properties of hexagonally arranged $\mathrm{Ni}$ nanowires embedded in anodic alumina templates. A particularly strong enhancement on their MO response, with respect to Ni bulk, was observed, which we attribute to a plasmon resonance effect. Changes induced in the spectra with changing $\mathrm{Ni}$ nanowire diameter were also studied.

The samples fabricated in this work were obtained by self-assembly using a two-step electrochemical aluminum anodization process. ${ }^{5}$ This anodic process leads to highly ordered alumina $\left(\mathrm{Al}_{2} \mathrm{O}_{3}\right)$ membranes with a so-called hexagonal two-dimensional polydomain arrangement of the pore channels. Using different anodization conditions, the size of the ordered pore domains, the interpore spacing, and the diameters of the pores can be controlled, the deviation in pore diameter being less than $10 \%$. The holes in the membrane were afterwards filled with nickel from aqueous electrolytes. The details of the sample preparation are given in Ref. 2. Two arrays of nanowires with different pore diameters, length, and interwire distance were prepared (see Fig. 1). The first sample (sample A) contains nanowires with diameter $d=35 \mathrm{~nm}$, length $L=1.4 \mu \mathrm{m}$ and interwire spacing $a=105$ $\mathrm{nm}$. This sample presents polydomains with long-range order of around $2.5 \mu \mathrm{m}$. The second sample (sample B) consists of Ni nanowire arrays with diameter $d=180 \mathrm{~nm}$, length $L=4$ $\mu \mathrm{m}$, and interwire distance $a=500 \mathrm{~nm}$. Such a sample shows hexagonally ordered domains of approximately $1.25 \mu \mathrm{m}$ in size. For Kerr measurements, the alumina template was fixed

\footnotetext{
${ }^{a}$ Electronic mail: sonia@imm.cnm.csic.es

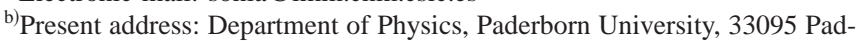
erborn, Germany.
}

to a $\mathrm{Si}$ substrate. The magnetic properties of these nanowire arrays have been previously characterized. ${ }^{3}$

Due to the nanowire geometry, a different optical response depending on the polarization direction of the incident light being along or perpendicular to the wire axis has been observed, ${ }^{6}$ leading to a different absorption spectra for the diagonal components of the dielectric tensor. In the same way, a different response is also expected between the MO components of the dielectric tensor $\left(\epsilon_{x y}, \epsilon_{x z}\right)$. Thus, we have measured the $\mathrm{MO}$ response of the prepared nanowire arrays in two different configurations (see top part of Fig. 2). In polar configuration, a magnetic field of $1.5 \mathrm{~T}$ is applied perpendicular to the sample plane $(X Y)$ along the wire axis $(z$ axis), the applied magnetic field being high enough to saturate the samples. The measured magnitude is the rotation $\phi_{k}$ of the plane of polarization of a linear polarized light reflected on the sample. The angle of incidence of light is $5^{\circ}$. For normal incidence, this magnitude depends on the $\epsilon_{x y}$ and $\epsilon_{x x}$ components of the dielectric tensor. In a transverse configuration, the magnetic field is applied in the plane of the sample and perpendicular to the plane of incidence, the angle of incidence being $45^{\circ}$. The applied field in this case $(0.35 \mathrm{~T})$ was not enough to reach magnetic saturation of the sample. The transverse Kerr data were corrected for saturation using the Kerr loops obtained from superconducting quantum in-
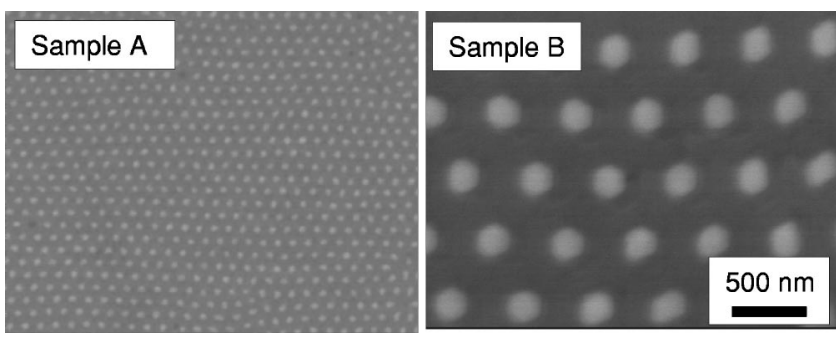

FIG. 1. Top-view scanning electron micrographs of nickel nanowires (light) embedded into the porous alumina matrix (dark). The scale is the same in both figures. 


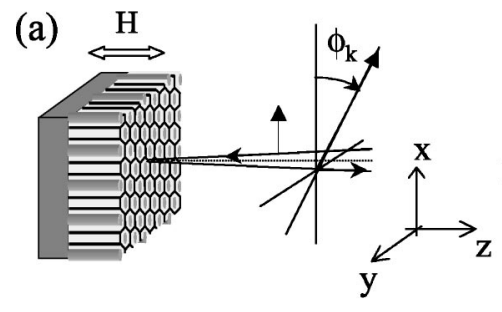

(b)
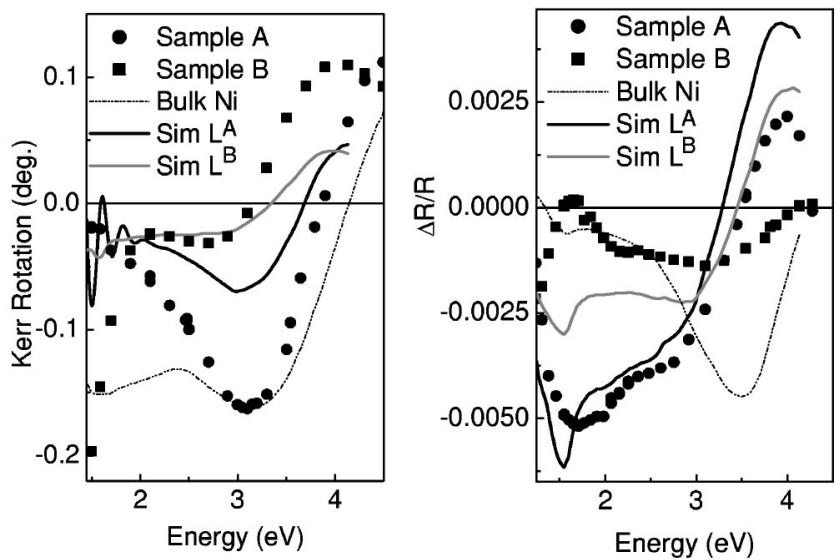

FIG. 2. MO polar (a) [transverse (b)] Kerr spectra for Ni nanowire arrays with different geometries: sample A: $35 \mathrm{~nm}$ diameter (circles), and sample B: $180 \mathrm{~nm}$ diameter (squares). Simulation with different shape factors (solid lines). Bulk Ni film (dashed lines).

terference device magnetometer measurements. ${ }^{3}$ The magnitude that we measure in transverse configuration is the variation of the reflectivity of the light polarized in the plane of incidence, due to the applied magnetic field, and normalized to the reflectivity at zero field $(\Delta R / R)$. This magnitude is related to the $\epsilon_{x z}, \epsilon_{x x}$, and $\epsilon_{z z}$ components of the dielectric tensor.

Figure 2 shows the MO spectra measured for the two Ni nanowire array samples (symbols). The spectra of the bulk Ni film (dashed line) taken from Ref. 7 has also been included for comparison. As can be observed the magnitude of the MO response $\left(\phi_{k}, \Delta R / R\right)$ of the Ni nanowire samples is very similar to that of a bulk $\mathrm{Ni}$ film. In particular, the polar rotation spectrum for sample A formed by nanowires with small diameter, $d=35 \mathrm{~nm}$, shows a strong peak around 3.1 $\mathrm{eV}$. This rotation peak has the same magnitude as the rotation peak of the bulk Ni sample although the amount of Ni contained in the nanowire sample is only around $15 \%$, so that an enhancement in the rotation around the peak position is observed when confining Ni in nanowire geometry.

In order to clarify the origin of the features found in these Kerr spectra, the dielectric tensor of the Ni nanowires layer, $\epsilon_{\mathrm{eff}}=\epsilon_{i j}^{\prime}+\mathrm{i} \epsilon_{i j}^{\prime \prime}$, has been computed in the same spectral range. In the case that the wire diameter is much lower than the wavelength of light, which is satisfied for sample A, the dielectric tensor can be modeled using an effective medium theory. ${ }^{8}$ In our case, a self-consistent approach described in detail in Ref. 9 was used to calculate the effective dielectric tensor. Thus, $\epsilon_{\text {eff }}^{\mathrm{A}}$ depends on the dielectric tensors of pure Ni and $\mathrm{Al}_{2} \mathrm{O}_{3}$, the amount of $\mathrm{Ni}$ in the alumina matrix, and on the nanowire's shape tensor $\Gamma^{\mathrm{A}}$, which is related to the depolarization tensor $\mathrm{L}^{\mathrm{A}}$ as $\Gamma^{\mathrm{A}}=-\mathrm{L}^{\mathrm{A}} / \epsilon_{\mathrm{eff}}^{\mathrm{A}}$. In the quasistatic approximation ${ }^{10}$ for cylinders whose axes are aligned with the principal axes, the diagonal components of the depolarDownloaded 14 May 2010 to 161.111.235.252. Redistribution subject

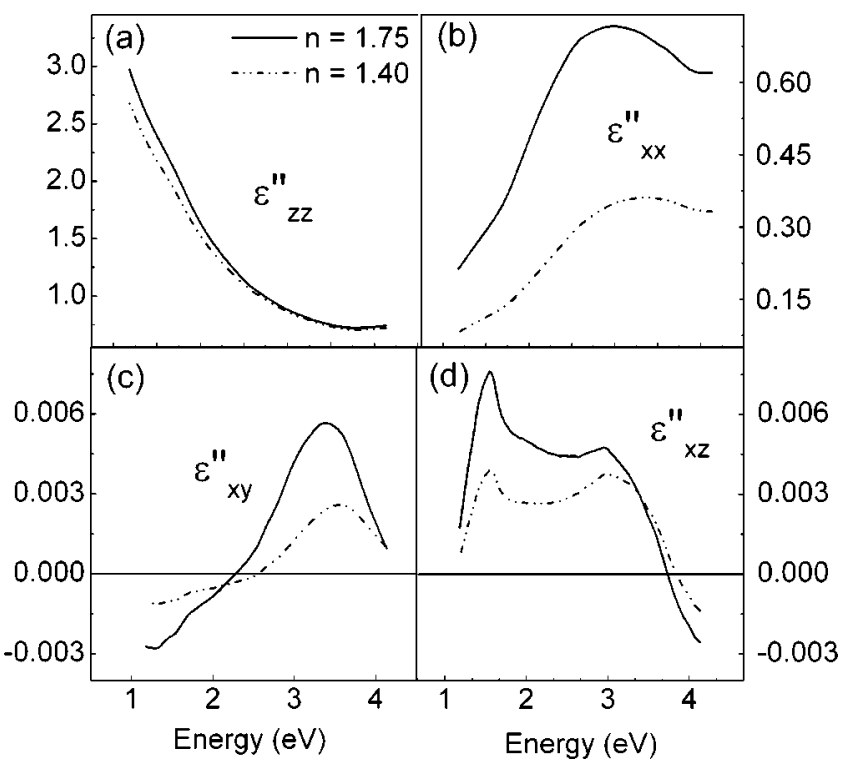

FIG. 3. Calculated values of the imaginary components of the effective dielectric tensor for the Ni nanowire layer embedded in two different templates: an alumina template with refractive index $n=1.75$ (solid lines); and a template with $n=1.40$ (dashed lines).

ization tensor takes the values $\mathrm{L}_{x x}^{\mathrm{A}}=\mathrm{L}_{y y}^{\mathrm{A}}=0.5$, and $\mathrm{L}_{z z}^{\mathrm{A}}=0$.

Figure 3 presents the calculated values of the imaginary components of the dielectric tensor for the nanowire layer as a function of energy. The spectra plotted with solid lines in Fig. 3 have been calculated considering the effective medium formed by $\mathrm{Ni}$ nanowires (Ni content 15\%) embedded in an alumina matrix whose refractive index is $n=1.75$. The refractive indices of bulk $\mathrm{Ni}$ were obtained from Ref. 11, whereas the MO coefficients of Ni were obtained from Ref. 12. The $\epsilon_{z z}^{\prime \prime}$ component of the nanowire layer [see Fig. 3(a)] shows a similar behavior to that observed for bulk $\mathrm{Ni}$, that is, decreasing in magnitude as the energy increases. In contrast, for light polarized perpendicular to the wires' axes, $\epsilon_{x x}^{\prime \prime}$, a strong absorption peak located at $2.6 \mathrm{eV}$ is observed [solid line in Fig. 3(b)]. We attribute this absorption peak to a plasmon resonance absorption. To support this assessment, we compare those spectra with the ones calculated for $\mathrm{Ni}$ nanowires embedded in a different template with refractive index $n=1.4$ (dashed lines in Fig. 3). (If the peak observed is related to a surface plasmon excitation due to the localization of the electromagnetic field at the wire surface, it will be very sensitive to the optical properties of the matrix. ${ }^{13}$ ) As expected, the peak observed in the $\epsilon_{x x}^{\prime \prime}$ component shifts from $2.6 \mathrm{eV}(n=1.75)$ to $3.0 \mathrm{eV}(n=1.40)$, which is the expected behavior for a plasmon resonant absorption [Fig. 3(b)]. In contrast, the $\epsilon_{z z}^{\prime \prime}$ component shows no appreciable difference for both templates, decreasing in magnitude as the energy increases.

For completeness, we also present the effect that the change in the dielectric constant of the matrix has on the nondiagonal components of the dielectric tensor [see Figs. 3(c) and 3(d)]: the peak observed around $3 \mathrm{eV}$ shifts to higher energies when decreasing the refractive index of the template, similar to the plasmon absorption peak. However, in the component $\epsilon_{x z}^{\prime \prime}$, no displacement of the peak located at $1.6 \mathrm{eV}$ is found [Fig. 3(d)]. This last feature is therefore not due to a plasmon excitation, and should be related to the to AIP license or copyright; see http://apl.aip.org/apl/copyright.jsp 
structure observed in the MO components of $\mathrm{Ni}^{12}$ We would like to point out that in contrast to the optical components, where a plasmon resonance is only observed when the polarization of the light is perpendicular to the wires axis (i.e., $\epsilon_{x x}$ or $\epsilon_{y y}$ components), the effect of the surface plasmon excitation is observed in all the nondiagonal components, although it is more important for $\epsilon_{x y}^{\prime \prime}$.

To directly compare this simulated effective dielectric tensor with the experimental results, we have computed the corresponding $\phi_{k}$ and $\Delta R / R$ Kerr MO spectra using a transfer matrix formalism. ${ }^{14}$ The simulation results are presented with a solid black line in Figs. 2(a) and 2(b). The simulated rotation spectrum reproduces qualitatively the experimental behavior, nicely, although a strong decrease of the value of the rotation peak is found with respect to the experimental data. This quantitative discrepancy on the intensity may be ascribed to a difference between the optical and/or MO coefficients of the electrodeposited $\mathrm{Ni}$ and those found in the literature. The simulated rotation spectrum presents oscillations in the infrared region due to optical interference effects between the Ni nanowire layer and the Si substrate. These oscillations are not present in the experimental spectra due to surface roughness or to residual absorption of the alumina matrix. The good agreement between the simulated and experimental rotation spectra confirms that the feature observed in the Kerr spectra is associated to the plasmon resonance of the nickel nanowires. In transverse configuration, the agreement with the simulated spectrum is quite remarkable, although the computed variation of the reflectivity for high energies (around $4 \mathrm{eV}$ ) exceeds the experimental value.

When increasing the wire diameter and interwire distance, for sample B $(d=180 \mathrm{~nm})$, marked changes occur on the shape of the MO spectra (see squares in Fig. 2). Although both samples contain the same amount of $\mathrm{Ni}$, around $15 \%$, the rotation spectra of samples A and B are different. The rotation curve of sample B is broadened and a shift of the spectrum to lower energies is observed, together with an increase of the rotation in the infrared region of the spectrum. Similarly, the shape of the transverse spectrum for sample B [squares in Fig. 2(b)] is quite different from the spectrum of sample A. Again, when the nanowire diameter increases the curve strongly broadens and decreases in magnitude.

The displacement observed for the plasmon resonance feature to lower energies when increasing the nanowire diameter has also been reported in $\mathrm{Au}$ and $\mathrm{Ag}$ nanostructures ${ }^{6,15,16}$ and is attributed to extrinsic particle size effects as, for example, phase retardation and dynamic depolarization. ${ }^{17,18}$ Such effects are not taken into account in a simple effective medium theory derived within the quasistatic limit (i.e., from consideration of the static electric field in the medium) where a static depolarization tensor is defined. For large particles of size comparable to wavelength, a more elaborated theory needs to be developed. However, a formal way to take into account the increase in the wire diameter is to consider a complex depolarization tensor, $\mathrm{L}$ $=\mathrm{L}_{\mathrm{dyn}}+\mathrm{iL}_{\mathrm{dam}}$, where the real part deviates from the static depolarization value due to dynamic depolarization effects that are the responsible of the shift of the plasmon resonance; the imaginary part accounts for the damping of the plasmons in the Ni nanowires by radiative losses, ${ }^{17,19,20}$ which increase as we increase the diameter of the wires. Thus, to simulate the MO spectra for sample B, we use a complex depolarization tensor $\mathrm{L}^{\mathrm{B}}$ for the wires: $\mathrm{L}_{x x}^{\mathrm{B}}=\mathrm{L}_{y y}^{\mathrm{B}}=0.4-\mathrm{i} 0.2$, and $\mathrm{L}_{z z}^{\mathrm{B}}=-\mathrm{i} 0.2$. The obtained spectra using these values are plotted with a solid gray line in Figs. 2(a) and 2(b). As can be observed, this simple model reproduces the tendency of the changes observed in the experimental spectra of one sample with respect to the other. For example, in the polar configuration, the rotation peak decreases in magnitude and the curve shifts to smaller energies, which is the observed experimental behavior. In the transverse configuration, there is a strong decrease in the intensity of the $\Delta R / R$ in the region between 1 and $3 \mathrm{eV}$ and a shift to higher energy of the crossing point at $3.4 \mathrm{eV}$, where the $\Delta R / R$ changes its sign. This is also the observed experimental behavior, although in this configuration, the experimental changes are stronger. A more elaborated model is under consideration.

In summary, the analyzed $\mathrm{Ni}$ nanowire arrays exhibit a high optical anisotropy, which leads to an anisotropy in their $\mathrm{MO}$ response. The MO activity of Ni nanowire arrays is strongly enhanced with respect to the bulk $\mathrm{Ni}$, making these systems interesting for possible MO applications. The MO spectra show a feature corresponding to the plasmon excitation of the nickel nanowires. This behavior has been reproduced by theoretical simulations of the spectra. The effect of increasing the nanowire diameter and interwire distance produces a strong plasmon resonance wavelength redshift, a decrease in its magnitude, and a strong broadening effect.

This work has partially been supported by The Ministry of Science and Technology (Spain) under Grant No. MAT2002-04484.

${ }^{1}$ M. Hwang, M. C. Abraham, T. A. Savas, H. I. Smith, R. J. Ram, and C. A. Ross, J. Appl. Phys. 87, 5108 (2000).

${ }^{2}$ K. Nielsch, F. Müller, A. P. Li, and U. Gösele, Adv. Mater. (Weinheim, Ger.) 12, 582 (2000).

${ }^{3}$ K. Nielsch, R. B. Wehrspohn, J. Barthel, J. Kischner, U. Gösele, S. F. Fischer, and H. Kronmüller, Appl. Phys. Lett. 79, 1360 (2001).

${ }^{4}$ Y. Peng, T.-H. Shen, and B. Ashworth, J. Appl. Phys. 93, 7050 (2003).

${ }^{5}$ H. Masuda and F. Fukuda, Science 268, 1466 (1995).

${ }^{6}$ J. R. Krenn, G. Schider, W. Rechberger, B. Lamprecht, A. Leitner, F. R. Aussenegg, and J. C. Weeber, Appl. Phys. Lett. 77, 3379 (2000).

${ }^{7}$ S. Visnovsky, V. Parizek, M. Nyvlt, P. Kielar, V. Prosser, and R. Krishnan, J. Magn. Magn. Mater. 127, 135 (1993).

${ }^{8}$ T. K. Xia, P. M. Hui, and D. Stroud, J. Appl. Phys. 67, 2736 (1989).

9 J. L. Menéndez, B. Bescós, G. Armelles, R. Serna, J. Gonzalo, R. Doole, A. K. Petford-Long, and M. I. Alonso, Phys. Rev. B 65, 205413 (2002).

${ }^{10}$ S. Berthier, J. Phys. I 4, 303 (1994).

${ }^{11}$ J. H. Weaver, C. Krafka, D. W. Lynch, and E. E. Koch, Phys. Daten. (1981).

${ }^{12}$ K. H. J. Buschow, Handbook of Magnetic Materials (Elsevier, Amsterdam, 1999).

${ }^{13}$ U. Kreibig and M. Vollmer, Optical Properties of Metal Clusters, Springer Series in Material Science Vol. 25 (Springer, Berlin, 1995).

${ }^{14}$ J. Zak, E. R. Moog, C. Liu, and S. D. Bader, Phys. Rev. B 43, 6423 (1991).

${ }^{15}$ Z. Liu, H. Wang, H. Li, and X. Wang, Appl. Phys. Lett. 72, 1823 (1998).

${ }^{16}$ G. Schider, J. R. Krenn, W. Gotschy, B. Lamprecht, H. Ditlbacher, A. Leitner, and F. R. Aussenegg, J. Appl. Phys. 90, 3825 (2001).

${ }^{17}$ M. Meier and A. Wokaun, Opt. Lett. 8, 581 (1983).

${ }^{18}$ M. Meier, A. Wokaun, and P. F. Liao, J. Opt. Soc. Am. B 2, 931 (1985).

${ }^{19}$ A. Wokaun, J. P. Gordon, and P. F. Liao, Phys. Rev. Lett. 48, 957 (1982).

${ }^{20}$ P. W. Barber, R. K. Chang, and H. Massoudi, Phys. Rev. Lett. 50, 997 (1983) 